\title{
Balancing high energy density and chemical stability in redox flow batteries with symmetric tetrazines.
}

\author{
Gloria D. De La Garza, a,b Aman Preet Kaur, ${ }^{\mathrm{b}, \mathrm{c}}$ Ilya A. Shkrob, b,d Lily A. Robertson,, ${ }^{\mathrm{b}, \mathrm{d}}$ Susan A. Odom, b,c,e \\ and Anne J. McNeil *a,b,f

\begin{abstract}
Nonaqueous redox flow batteries are a promising technology for grid-scale energy storage, however, their commercial success relies on identifying redox active materials that exhibit extreme potentials, high solubilities in all states of charge, and long cycling stabilities. Meeting these requirements has been particularly challenging for molecules capable of storing negative charge. Within this context, the symmetric tetrazines remain unexplored despite their unique structural properties that enable them to meet these challenges. Herein, we prepared s-tetrazines substituted with methyl, methoxy, and thiomethyl substituents and evaluated their electrochemical properties, solubility, and cycling stability. These studies revealed that 3,6-dimethoxy-s-tetrazine undergoes a reversible one-electron reduction to generate a soluble ( $>0.5 \mathrm{M}$ in electrolyte/solvent) and stable $\left(t_{1 / 2}>1240 \mathrm{~h}\right)$ radical anion. When implemented in a lab-scale flow battery, it exhibited a relatively slow capacity fade of $13 \%$ over 100 cycles $(38 \mathrm{~h})$. Given their uncommonly high solubility and cycling stability, we believe that $s$-tetrazine derivatives should be further explored for non-aqueous redox flow batteries.
\end{abstract}

\section{Introduction}

Replacing carbon pollution-generating energy sources with renewable energy sources is essential to reduce our greenhouse gas emissions and meet our targets. ${ }^{1}$ However, the intermittent nature of solar and wind energy production due to seasonal and daily cycles poses a major challenge. ${ }^{2}$ Consequently, large-scale energy storage technologies are needed to prevent interruptions caused by these cycles. ${ }^{3}$ In this context, redox flow batteries (RFB) have emerged as a promising energy storage technology because, unlike traditional batteries, the power and capacity can be scaled independently by changing the electrode surface area (power) and/or the volume and concentration of electroactive species (capacity).4,5,6 As a result, RFBs can be tailored to variously sized applications, including integration into the electrical grid and homes.

Redox flow batteries store charge within redox-active molecules via oxidation and reduction reactions. Most commercial RFBs use inorganic species ${ }^{7}$ such as vanadium, ${ }^{8,9}$ iron-chromium, ${ }^{10}$ or zinc-cerium salts, ${ }^{11}$ which are dissolved in aqueous electrolytes. However, these systems face several limitations, including low operating voltages (1.1-1.6 V), ${ }^{4}$ highcost materials, ${ }^{12,13}$ and corrosive supporting electrolytes (e.g., 5 $\left.\mathrm{M} \mathrm{H}_{2} \mathrm{SO}_{4}\right) .{ }^{7}$ For these reasons, redox flow batteries using nonaqueous solvents and redox-active organic molecules are being explored. ${ }^{14,15,16,17}$ Non-aqueous redox flow batteries (NRFBs) have a wider window of electrochemical stability (up to

\footnotetext{
a. Department of Chemistry, University of Michigan, 930 North University Avenue,

Ann Arbor, Michigan, 48108-1055, United States

b. Joint Center for Energy Storage Research (JCESR), 9700 South Cass Avenue, Lemont, Illinois, 60439, United States

Department of Chemistry, University of Kentucky, 505 Rose Street, Lexington, Kentucky, 40506, United States

d. Chemical Sciences and Engineering Division, Argonne National Laboratory, 9700

South Cass Avenue, Lemont, Illinois 60439, United States

e. Deceased.

f. Macromolecular Science and Engineering Program, University of Michigan, 2800

Plymouth Road, Ann Arbor, Michigan, 48109-2800, United States
}

$5 \mathrm{~V}$ in acetonitrile), enabling access to larger battery voltages. In addition, redox-active organic molecules can be synthetically tuned to achieve high energy densities, solubilities, and stabilities. Moreover, organic molecules are composed of earthabundant elements, leading to inexpensive ${ }^{12}$ and potentially sustainably sourced charge-storage materials.

To date, many organic molecules have been evaluated as anolytes (negative charge carriers) and catholytes (positive charge carriers) in NRFBs. ${ }^{14,15,16,17}$ Among the anolytes, $\mathrm{N}$ heterocyclic aromatic compounds have been prominent due to their reversible reductions, including viologens, ${ }^{18,19,20,21,22,23} \mathrm{~N}$ substituted phthalimides, $24,25,26,27,28,29,30 \quad 2,1,3-$ benzothiadiazoles, $31,32,33,34$ pyridiniums, $35,36,37,38,39,40$ and bipyrimidines. ${ }^{41}$ Despite these successes, the structural variety within these anolytes is limited due to the stringent requirements posed by the application (i.e., combining low redox potentials and high solubility while being exceptionally stable in all states of charge). Therefore, a new addition to this short list will advance the state of art.

Our key insight herein is recognizing that 1,2,4,5-tetrazines ( $s$ tetrazines, Tz) might be an even better anolyte based on their (i) exceptional chemical stability, (ii) high solubility in all states of charge, and (iii) low molar mass. With respect to chemical stability, some anolytes with low reduction potentials yield unstable radical anions because they are strong bases and deprotonate the solvent and/or react with protic impurities, resulting in gradual capacity fade. ${ }^{32,42}$ We hypothesized that $s$ tetrazines might be less basic due to the delocalized charge distribution across all four nitrogens. With respect to solubility, low molecular weight anolytes are often not sufficiently soluble in polar solvents used in flow batteries due to favourable $\pi$ stacking, ${ }^{43}$ which results in aggregation and precipitation. Therefore, large, bulky moieties are often added to improve anolyte solubility but due to their high molecular weight they cannot attain the high concentrations required for NRFBs to become competitive with other energy storage devices. Other creative approaches to solve this problem include adding ionic 
moieties to attenuate $\pi$-stacking through electrostatic repulsion or introducing $\mathrm{H}$-bonding interactions to outcompete $\pi$ stacking. ${ }^{33} \mathrm{~A}$ most unusual property of $s$-tetrazines is that due to $\pi$-orbital symmetry, $\pi$-stacking is not favourable (based on crystal structures $\left.{ }^{44,45,46}\right)$. As a result, we predicted that $s$ tetrazines would exhibit high solubility in polar solvents even as low molar mass materials. In addition to these considerations, $s$-tetrazines are easy to access synthetically and have potential end-of-life reuse by other industries, making them an appealing choice for NRFBs.

s-Tetrazines (< 120-200 Da) are already known for their reversible electrochemical reductions and used in diverse applications, ${ }^{47}$ including as electro(fluoro)chromic materials, ${ }^{48,49}$ photocatalysts, ${ }^{50,51,52,53,54}$ and components for solid-state batteries. ${ }^{55,56}$ Here we explore their potential for redox flow batteries. More specifically, we evaluate three symmetrically substituted derivatives with methyl (Tz-Me), methoxy (Tz-OMe), and thiomethyl (Tz-SMe) groups. Among these derivatives, Tz-OMe demonstrated both high solubility and long cycling stability. Moreover, a high intrinsic capacity (charge stored per gram of material) can be reached given its low molar mass. Due to these favourable properties, our studies suggest that $s$-tetrazine derivatives deserve more attention in the energy storage community.
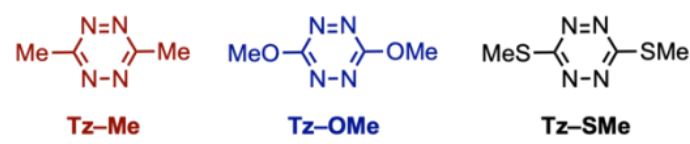

\section{Results and discussion}

Syntheses and (spectro)electrochemical characterization. sTetrazines can be purchased (Tz-SMe) or synthesized in a onepot procedure from commercial reagents in low (Tz-Me, 29\%) ${ }^{57}$ to high (Tz-OMe, 92\%) ${ }^{58}$ isolated yields (SI pp. S4-S5). The low isolated yield in the Tz-Me synthesis is attributed to unintended sublimation of the product during solvent removal. In contrast, Tz-OMe does not easily sublime and can be purified by column chromatography. Compound identities and purities were supported by high-resolution mass spectrometry, ${ }^{1} \mathrm{H}$ and ${ }^{13} \mathrm{C}$ nuclear magnetic resonance spectroscopies, and elemental analysis (SI pp. S4-S8).

To determine the reduction potential and electrochemical reversibility, cyclic voltammetry (CV) was performed in $0.5 \mathrm{M}$ TBAPF $_{6}$ (tetra( $n$-butyl)ammonium hexafluorophosphate) in acetonitrile (Figure $1 \mathrm{~A}$ ). These voltammograms revealed a reversible one-electron reduction of the $s$-tetrazine with halfwave potentials of $-1.40 \mathrm{~V}(\mathrm{Tz}-\mathrm{Me}),-1.20 \mathrm{~V}(\mathrm{Tz}-\mathrm{OMe})$, and $1.11 \mathrm{~V}(\mathrm{Tz}-\mathrm{SMe})$ relative to the ferrocene/ferrocenium couple. These low reduction potentials are similar to other anolytes explored for nonaqueous RFBs ${ }^{14}$ and can result in a high voltage battery (>2 V) when paired with an appropriate catholyte. The relatively small differences in potential between the methyl, thiomethyl, and methoxy derivatives are consistent with previous reports that showed a minimal impact of the substituent identity (for $\mathrm{C}, \mathrm{O}$, and $\mathrm{S}$-based substituents) on tetrazine reduction potentials. ${ }^{59,60}$ These results were rationalized based on the fact that the lowest unoccupied molecular orbitals (LUMO) are primarily localized on the central ring with negligible contributions from the substituents. ${ }^{59}$

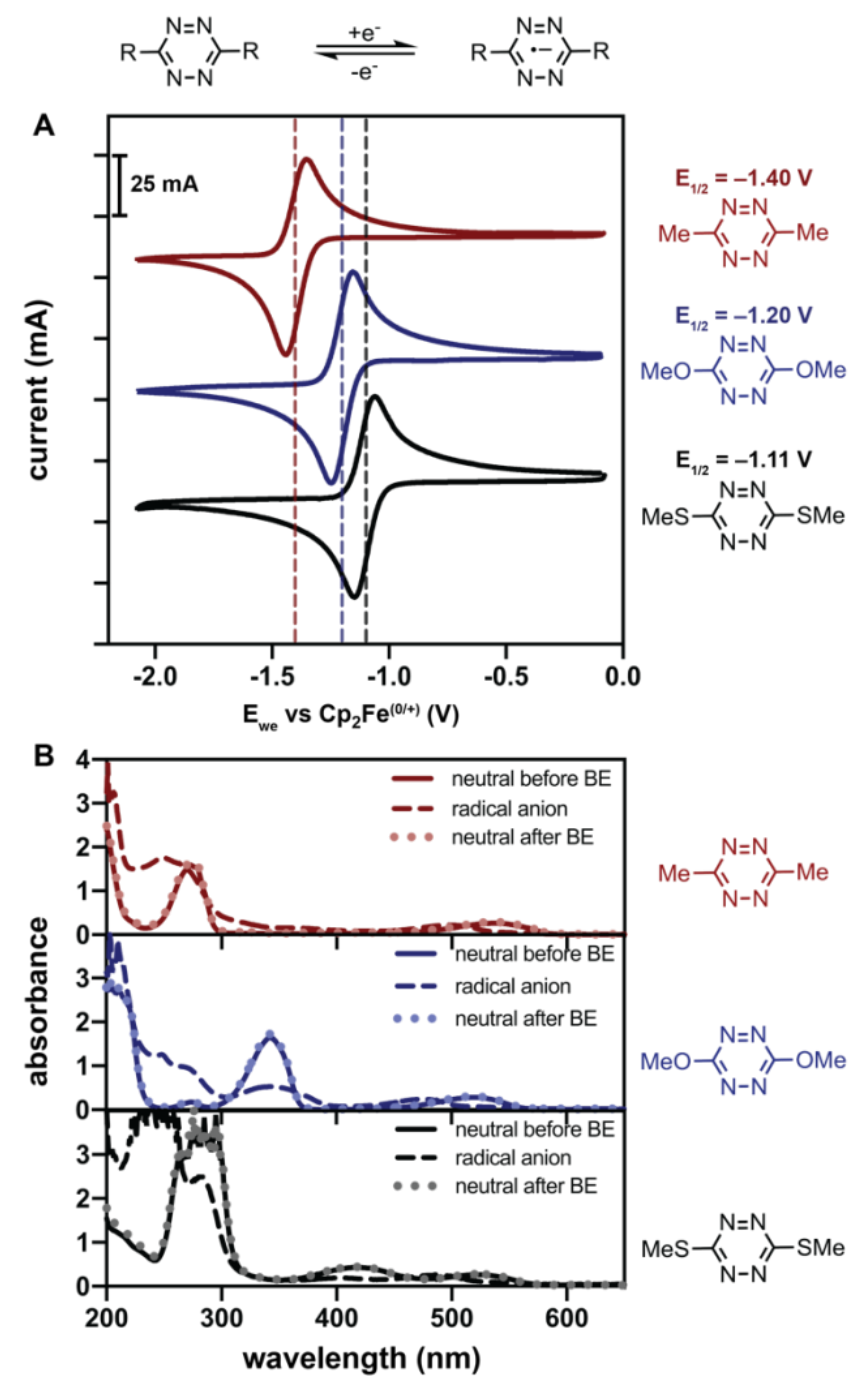

Figure 1. (A) Cyclic voltammograms of one-electron reduction of $5 \mathrm{mM}$ Tz-Me (top), Tz-OMe (middle), and Tz-SMe (bottom) in $0.5 \mathrm{M} \mathrm{TBAPF}_{6}$ in acetonitrile at a scan rate of $10 \mathrm{mV} / \mathrm{s}$. (B) UV/vis spectra of the neutral tetrazines (2.5 mM with 0.5 $\mathrm{M} \mathrm{TBAPF}_{6}$ in acetonitrile) before bulk electrolysis (BE) reduction (solid line), as the radical anion (dashed line), and after bulk electrolysis re-oxidation to form the neutral tetrazine (dotted line).

To determine whether the one-electron reduction of these $s$ tetrazine derivatives was reversible, the ratios of cathodic and anodic peak currents were measured. Redox couples involving chemically stable species generally have a peak ratio close to one, regardless of the scan rate. ${ }^{61}$ For all three tetrazines studied herein, the peak ratios were found to be approximately $1( \pm 0.1)$ for all scan rates examined $(5-500 \mathrm{mV} / \mathrm{s})$, indicating chemically reversible reductions (SI pgs S16-S17). 
Electrochemical reversibility was also evaluated by calculating the peak-to-peak separations, which were both scan-rate dependent and broader than the ideal value $(59 \mathrm{mV})$, indicating a quasi-reversible electrochemical reduction.

To further investigate reversibility on a longer timescale, spectroelectrochemical studies were performed by acquiring UV-vis absorption spectra in dilute solutions (Figure 1B, solid lines; SI pp. S27-S28). All three neutral tetrazines exhibited a low-energy absorption in the visible region, attributed to an $n \rightarrow \pi^{*}$ transition that is weakly dependent on the substituent identity. A second, more intense $\pi \rightarrow \pi^{*}$ absorption is also observed, which is more strongly impacted by the substituent identity. Upon reduction, significant blue shifts in the absorption onsets are observed for all three tetrazines (Figure 1B, dashed lines). Gratifyingly, upon re-oxidation, the absorption profiles of the neutral species were once again observed (Figure 1B, dotted lines), suggesting the redox processes are reversible for all three derivatives under these conditions.

After observing both chemical and electrochemical reversibility on these longer timescales, we next sought to determine the diffusion coefficient (D) and heterogeneous electron-transfer rate constant $\left(k_{0}\right)$, which must be fast to access high current densities and minimize detrimental overpotentials. The Randles-Sevcik equation ${ }^{61}$ was used to calculate diffusion coefficients, which describe the mass transport of the redoxactive species from the bulk solution to the electrode. The estimated diffusion coefficients for the neutral tetrazines were similar to each other $\left(1.4-1.6 \times 10^{-5} \mathrm{~cm}^{2} / \mathrm{s}\right)$ and on par with other anolytes used in redox flow batteries (e.g., $8.4 \times 10^{-6}$ $\mathrm{cm}^{2} / \mathrm{s}$ for $N$-methylphthalimide ${ }^{62}$ and $1.9 \times 10^{-5} \mathrm{~cm}^{2} / \mathrm{s}$ for $2,1,3-$ benzothiadiazole ${ }^{31}$ ). In addition, a linear correlation between the current and the square-root of the scan rate was observed, further supporting a diffusion-limited chemically reversible process (SI pp. S17-S19). ${ }^{61}$ Next, the heterogeneous electrontransfer rate constant, which describes how fast the redoxactive species undergoes a reaction at the electrode surface, was calculated using the Nicholson method. ${ }^{63}$ The electrontransfer rate constants for the $s$-tetrazines were again similar to each other (estimated to be $3-4 \times 10^{-3} \mathrm{~cm} / \mathrm{s}$; SI pp. S18-S19), and on par with similar anolytes in the literature (e.g., $2.5 \times 10^{-}$ ${ }^{3} \mathrm{~cm} / \mathrm{s}$ for $\mathrm{N}$-methylphthalamide ${ }^{62}$ and $0.9 \times 10^{-2} \mathrm{~cm} / \mathrm{s}$ for $2,1,3-$ benzothiadiazole ${ }^{31}$ ), supporting their viability in RFBs.

Stability during cycling. Given the electrochemical reversibility observed in the CV studies above, we began by evaluating all three $s$-tetrazines in galvanostatic charge-discharge cycling to assess their long-term stability under dilute conditions ( $5 \mathrm{mM}$ ). Cycling was performed between the reduced and neutral anolyte in an $\mathrm{H}$-cell (Figure $2 \mathrm{~A}$ ) and the discharge capacity was monitored as a function of time. In addition, the chargedischarge profiles were monitored to ensure the cycling was going through the intended tetrazine redox couple $\left(\mathrm{Tz} / \mathrm{Tz}^{\bullet-}\right)$ and not through degradation products. Here we observed significant variations in stability as a function of substituent identity (Figure 2B). For example, the Tz-Me showed the largest capacity loss ( $97 \%$ over $1.4 \mathrm{~h}$ ), followed by Tz-SMe (32\% over $13 \mathrm{~h})$. In contrast, Tz-OMe exhibited a negligible capacity loss ( $3 \%$ over $26 \mathrm{~h}$ ). Cyclic voltammograms acquired before and after these cycling experiments were consistent with the capacity fades observed during cycling, with $80 \%$ loss in peak current for $\mathrm{Tz}-\mathrm{Me}$, a $40 \%$ loss for Tz-SMe, and a 4\% loss for the Tz-OMe (SI Figures S20-S21). These large losses in current are indicative of chemical decomposition and/or precipitation. ${ }^{64}$ In contrast, the negligible capacity fade and current loss for Tz-OMe suggests that it may be a promising anolyte for RFBs, and consequently, all remaining studies were performed with Tz-OMe.

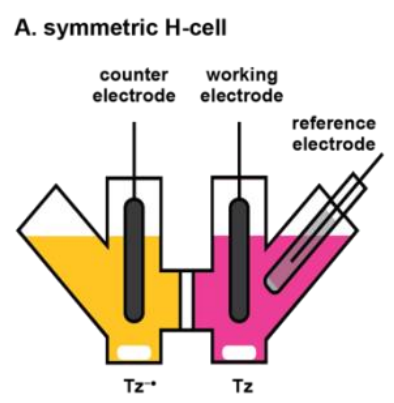

C. electrolyte screen

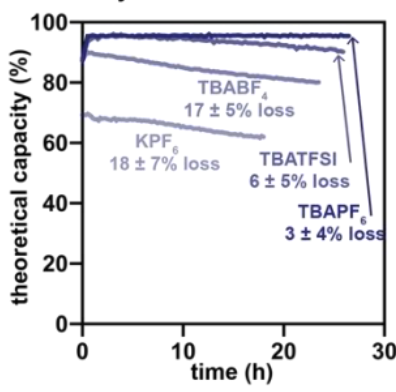

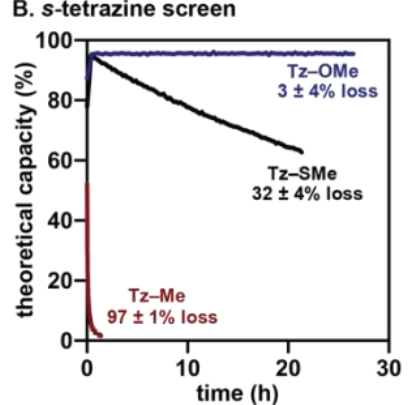

D. solvent screen

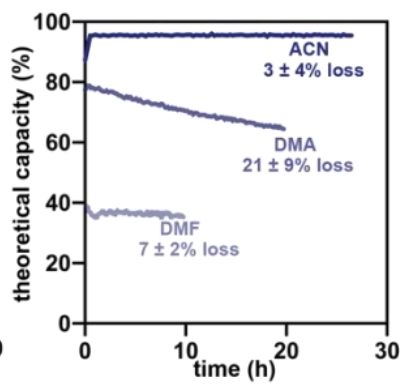

Figure 2. (A) Symmetric $\mathrm{H}$-cell cycling between reduced $\left(\mathrm{Tz}^{-\bullet}\right)$ and neutral $(\mathrm{Tz})$ species. In each case, the percent loss is calculated relative to the first cycle. (B-D) Plots of the theoretical discharge capacity versus time for galvanostatic charge-discharge cycling experiments. (B) Screening $5 \mathrm{mM}$ s-tetrazine derivatives Tz-OMe (blue), Tz-SMe (black), Tz-Me (red) in $0.5 \mathrm{M} \mathrm{TBAPF}_{6}$ in acetonitrile. (C) Screening electrolytes for $5 \mathrm{mM} \mathrm{Tz-OMe} \mathrm{with} 0.5 \mathrm{M} \mathrm{TBAPF}_{6}$ (dark blue), TBATFSI (medium blue), $\mathrm{TBABF}_{4}$ (blue), and $\mathrm{KPF}_{6}$ (light blue) in acetonitrile. (D) Screening solvents for $5 \mathrm{mM} \mathrm{Tz-OMe} \mathrm{in} \mathrm{acetonitrile} \mathrm{(ACN,}$ dark blue), dimethylacetamide (DMA, medium blue), dimethylformamide (DMF, light blue) with $0.5 \mathrm{M} \mathrm{TBAPF}_{6}$.

Optimizing Cycling Conditions. Because supporting electrolyte and solvent can have a large impact on cycling stability, in part due to undesired reactivity with radical anions, we next sought to optimize the electrolyte and solvent combination for $\mathrm{Tz}-$ OMe. To accomplish this goal, Tz-OMe was subjected to galvanostatic charge-discharge cycling under different conditions (SI pp. S21-S22). First, four different supporting electrolytes were evaluated (Figure 2C). Three electrolytes had the same cation $\left(\mathrm{TBA}^{+}\right)$but different anions (hexafluorophosphate $\left(-\mathrm{PF}_{6}\right)$, tetrafluoroborate $\left(-\mathrm{BF}_{4}\right)$ and bistrifluoromethanesulfonimide (-TFSI)). The fourth electrolyte had the same anion $\left(-\mathrm{PF}_{6}\right)$ but a different cation $\left(\mathrm{K}^{+}\right.$versus $\left.\mathrm{TBA}^{+}\right)$. Most electrolytes exhibited small losses per cycle after the first few cycles, which suggests that there were some reactive 
impurities that were consumed. The $\mathrm{TBAPF}_{6}$ outperformed the other electrolytes, and was chosen for all future studies based on the smallest capacity fade after 100 cycles ( $26 \mathrm{~h}$ ).

Next, two additional solvents with different dynamic viscosities $(\mu)$ and relative dielectric permittivities $(\varepsilon)$ were evaluated (SI pp. S21-S22). Lower solvent viscosities often lead to higher ion conductivities (positive impact) whereas lower relative permittivities lead to lower solubilities of supporting electrolyte and redox-active species (negative impact). Bulk electrolysis cycling of Tz-OMe in acetonitrile ( $\mu=0.34 \mathrm{cP}, \varepsilon=35.9), \mathrm{N}, \mathrm{N}$ dimethylformamide (DMF, $\mu=0.80 \mathrm{cP}, \varepsilon=36.7$ ), and $N, N$ dimethylacetamide (DMA, $\mu=5.2 \mathrm{cP}, \varepsilon=176$ ) was performed (Figure 2D). ${ }^{65}$ Both DMF and acetonitrile, which have similar properties, performed similarly after the first few cycles. However, DMF exhibited a capacity loss initially, again suggesting a reactive impurity that was consumed. All things considered, these studies suggest that acetonitrile was the best performing solvent for this anolyte/supporting electrolyte combination and was therefore used in all subsequent experiments.

Solubility measurements. The solubility of the redox active species impacts the RFB capacity because more charge can be stored in a given volume when the solubility is higher. Solubilities exceeding $1 \mathrm{M}$ (in all charge states) are targeted to be competitive with the commercial vanadium RFBs. ${ }^{14}$ Therefore, the solubility of Tz-OMe was measured using ${ }^{1} \mathrm{H}$ NMR spectroscopy of saturated solutions (SI pp. S10-S15). In the neutral state, Tz-OMe exhibits an impressive solubility of 2.3 $\mathrm{M}$ in acetonitrile. For comparison, 2,1,3-benzothiadiazole, which is one of the most soluble anolytes known, has a solubility of $5.7 \mathrm{M}$ in acetonitrile. ${ }^{31}$ In contrast, anolytes like $\mathrm{N}$ methylphthalimide ${ }^{26}$ have a much lower solubility $(0.5 \mathrm{M}$ in propylene carbonate). ${ }^{19}$ Although the Tz-OMe solubility is high in acetonitrile, it is important to also measure the solubility with the supporting electrolyte used in the redox flow batteries present. Typically, the solubility decreases with added supporting electrolyte because the solvent's polarity has increased. Indeed, the solubility of Tz-OMe decreases to 0.79 $\mathrm{M}$ with $0.79 \mathrm{M} \mathrm{TBAPF}_{6}$ in acetonitrile, which is again about half as soluble as 2,1,3-benzothiadiazole $(2.1 \mathrm{M}$ with $2.1 \mathrm{M}$ supporting electrolyte in acetonitrile) but still close to the target of $1 \mathrm{M}^{31}$

As noted above, the solubility of the molecule in each state of charge should exceed $1 \mathrm{M}$. Unfortunately, all attempts at measuring the solubility of the Tz-OMe radical anion were unsuccessful. For example, treating Tz-OMe with a chemical reductant ( $\mathrm{Na}$ metal) led to a challenging separation and isolation. In addition, efforts to isolate the radical anion after electrochemical reduction also failed. Nevertheless, as described later, the fact that we were able to run a RFB at 0.5 $\mathrm{M}$ Tz-OMe demonstrates that the radical anion (with TBA ${ }^{+}$ counterion) is at least that soluble. Future studies will explore eutectic mixtures and side-chain modification to further improve the solubilities.
Radical anion characterization. Given the negligible losses in capacity during galvanostatic charge-discharge cycling over 26 $\mathrm{h}$ (indicating good cycling stability), we next evaluated calendar lifetime, which refers to the lifetime of a charged species in the bulk (e.g., in a storage tank). ${ }^{42}$ To do this, we used electron paramagnetic resonance spectroscopy (EPR) to both characterize the radical anion and quantitatively determine its lifetime (SI pp. S29-S30). The radical anion was first generated electrochemically and then observed using a continuous-wave $X$-band EPR spectrometer. The spectrum of the radical anion is a set of nine equidistant lines originating from four magnetically equivalent ${ }^{14} \mathrm{~N}$ nuclei in the $s$-tetrazine ring (SI Figure S30). The EPR spectra were then collected at constant time intervals over $200 \mathrm{~h}$. Around $90 \mathrm{~h}$, oxygen breached into the sample and began quenching the radical anion. By fitting the decay before the breach, we calculated a half-life of $\sim 1240 \mathrm{~h}$ in $0.5 \mathrm{M} \mathrm{TBAPF}_{6}$ in acetonitrile, which exceeds that of 2,1,3-benzothiadiazole ( $~ 852 \mathrm{~h})$ in the same electrolyte/solvent combination. Overall, this remarkable stability is competitive with some of the best anolytes examined to date. ${ }^{14}$

Flow battery evaluation. Given the long cycling and calendar stability, we next evaluated Tz-OMe in a laboratory-scale flow battery. To identify a compatible catholyte, sequential CV scans of a 1:1 mole ratio of Tz-OMe and the catholyte were overlaid (SI Figure S22). Because no changes were observed in the peak currents nor any evidence of new species, we concluded that all four catholytes were compatible with Tz-OMe. From this group, 1,4-di-tert-butyl-2,5-bis(2-methoxyethoxy)benzene (DBBB) was selected for further studies due to its high oxidation potential, leading to a battery voltage of $1.87 \mathrm{~V}$ (recall that the technoeconomic target for RFBs is $>2 \mathrm{~V}$ ), and its commercial availability. Next, we tested the performance of the mixed electrolyte in a flow battery using a porous membrane, which has high ionic conductivity but poor selectivity, resulting in crossover of both the electrolyte and redox active species. To attenuate the detrimental impacts of active species crossover, a symmetrical setup with $50 \mathrm{mM}$ of both the anolyte and catholyte in both reservoirs was used (Figure $3 \mathrm{~A}$ ). These electrolyte solutions were flowed through the cell for galvanostatic charge-discharge cycling at $12 \mathrm{~mA} / \mathrm{cm}^{2}(2.6 \mathrm{C})$ and up to $80 \%$ state of charge. A small loss in capacity (13\%) was observed over $38 \mathrm{~h}$ (100 cycles; Figures $3 \mathrm{~B}$ and $3 \mathrm{C}$ ). Post-run CV analysis showed an $8 \%$ loss in concentration for Tz-OMe (anolyte) and 4\% for DBBB (catholyte), respectively, consistent with the capacity losses observed during cycling (SI Figure S25). The post-run $\mathrm{CV}$ analysis also showed a small irreversible oxidation at $0.1 \mathrm{~V}$ versus $\mathrm{Cp}_{2} \mathrm{Fe}^{(0 /+)}$ indicative of some degradation during cycling.

Encouraged by these results, we next ran a flow battery under more realistic conditions using a higher concentration of both Tz-OMe and DBBB (0.5 M with 0.5 $\mathrm{M} \mathrm{TBAPF}_{6}$ in acetonitrile). The same porous membrane was utilized but a higher charging rate $\left(42 \mathrm{~mA} / \mathrm{cm}^{2}, 1 \mathrm{C}\right)$ was used to minimize capacity fade due to crossover of charged species. The galvanostatic charge- 
discharge cycling over $15 \mathrm{~h}$ (15 cycles) showed a 6\% capacity fade (SI Figure S26). These results compare favourably to a similar flow battery using $0.5 \mathrm{M}$ 2,1,3-benzothiadiazole and a structural analogue of DBBB, wherein an approximately $25 \%$ capacity fade was observed over $24 \mathrm{~h}$ (22 cycles). ${ }^{31}$ In both cases, the materials utilization was low ( $55 \%$ for $\mathrm{Tz}-\mathrm{OMe}$ and $\sim 30 \%$ for the 2,1,3-benzothiadiazole), potentially due to viscous solutions. Post-run CV analysis of the anolyte reservoir revealed a $46 \%$ loss in peak current for the Tz-OMe and no loss in the peak current for DBBB. These results are consistent with precipitation, perhaps due to the presence of $0.5 \mathrm{M}$ DBBB, which was not included in the solubility studies. Nevertheless, slow capacity fade in the high concentration flow battery places Tz-OMe among the best anolytes in nonaqueous flow batteries.

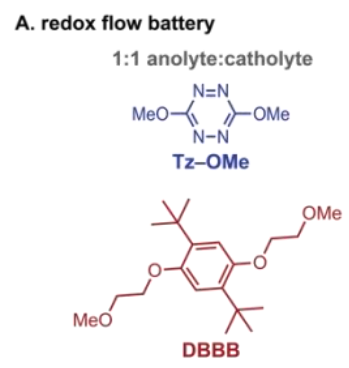

B. capacity and efficiency over time

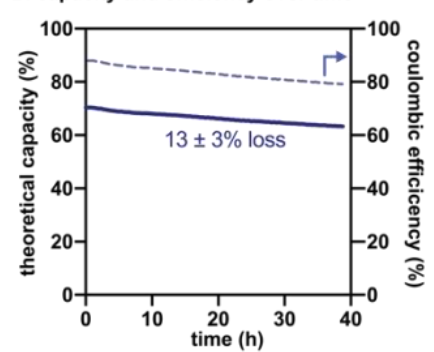

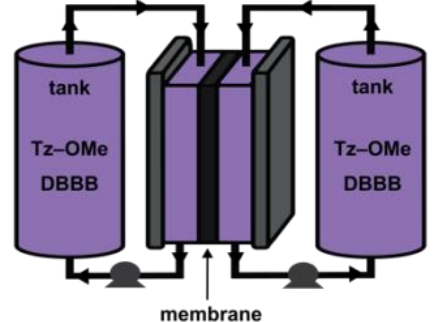

C. charge/discharge cycles

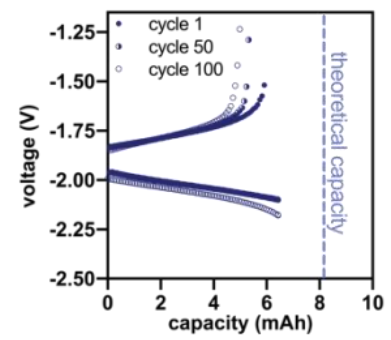

Figure 3. (A) RFB diagram with $1: 1$ combination of $50 \mathrm{mM}$ anolyte (Tz-OMe) to $50 \mathrm{mM}$ catholyte (DBBB) in $0.5 \mathrm{M} \mathrm{TBAPF}_{6}$ in acetonitrile. (B) Plot of theoretical discharge capacity and coulombic efficiency versus time for galvanostatic chargedischarge cycling. (C) Plot showing charge-discharge profiles for cycle 1,50 , and 100 .

\section{Conclusion}

In this study, we inquired whether the unique structural features of $s$-tetrazines make them suitable as anolytes in NRFBs. To this end, three centrosymmetric tetrazine derivatives with different substituents (-Me, -OMe, and -SMe) were synthesized and evaluated. All three derivatives were electrochemically reversible, however, the Tz-OMe derivative emerged as the most promising anolyte based on cycling stability. Further studies revealed that the radical anion of this molecule has a half-life $>10^{3} \mathrm{~min}$, which makes this system competitive with the best organic anolytes currently used in the field. A laboratory-scale flow battery test using $50 \mathrm{mM} \mathrm{Tz-OMe}$ and a common catholyte showed a mild capacity fade ( $13 \%$ over 100 cycles). Further studies with a 10-fold higher concentration were similarly stable. Even though Tz-OMe has exceptionally high solubility in acetonitrile and acetonitrile based electrolytes, it can be further increased (in future studies) through electrolyte optimization, and might eventually reach the high energy density required by technoeconomic models. Overall, our results suggest that $s$-tetrazine-based molecules exemplify a new strategy for balancing the high energy density and chemical stability, making them a welcome addition to the select few classes of $\mathrm{N}$-heterocyclic anolytes.

\section{Author Contributions}

AJM and GDD conceptualized the idea. AJM and GDD led the team in project planning and experimental design. GDD synthesized and characterized organic compounds, conducted electrochemical characterization and redox flow battery studies, measured solubility, validated and curated data, performed formal data analysis, and created data visualization graphics. APK performed spectroelectrochemical characterization, data curation, and formal data analysis of that data. SAO connected GDD with APK. IAS and LAR performed the electron paramagnetic spectroscopy experiments, performed the data analysis, and created the data visualization graphics. AJM and GDD wrote the original draft. All authors discussed experimental results and reviewed and edited the manuscript. All authors gave final approval for publication.

\section{Acknowledgements}

This paper is dedicated to the memory of Susan A. Odom, a fierce and creative scientist, and cherished member of the JCESR team. This research was supported by the Department of Energy through the Joint Center for Energy Storage Research (JCESR), an Energy Innovation Hub funded by the U.S. Department of Energy, Office of Science, Basic Energy Sciences. GDD thanks the National Science Foundation for a Graduate Research Fellowship. The authors thank Prof. Melanie Sanford (and the Sanford group) for helpful discussions, and Jessica L. Tami for performing the catholyte compatibility experiments. The submitted manuscript has been created by UChicago Argonne, LLC, Operator of Argonne National Laboratory ("Argonne"). Argonne, a U.S. Department of Energy Office of Science laboratory, is operated under Contract No. DEAC02-06CH11357. The U.S. Government retains for itself, and others acting on its behalf, a paid-up nonexclusive, irrevocable worldwide license in said article to reproduce, prepare derivative works, distribute copies to the public, and perform publicly and display publicly, by or on behalf of the Government.

\section{Notes and references}


1 The long-term strategy of the United States: Pathways to netzero greenhouse gas emissions by 2050, https://www.whitehouse.gov/wp-content/uploads/2021/10/USLong-Term-Strategy.pdf, (accessed Dec 9, 2021).

2 P. Das, J. Mathur, R. Bhakar, and A. Kanudia, Implications of short-term renewable energy resource intermittency in longterm power system planning. Energy Strategy Rev., 2018, 22, 115.

${ }^{3}$ T. M. Gur, Review of electrical energy storage technologies, materials and systems: challenges and prospects for large-scale grid storage. Energy Environ. Sci., 2018, 11, 2696-2767.

${ }^{4}$ P. Arévalo-Cid, P. Dias, A. Mendes, and J. Azevedo, Redox flow batteries: a new frontier on energy storage. Sustain. Energy Fuels, 2021, 5, 5366-5419.

${ }^{5}$ E. Sanchez-Díez, E. Ventosa, M. Guarnieri, A. Trovò, C. Flox, R. Marcilla, F. Soavi, P. Mazur, E. Aranzabe, and R. Ferret, Redox flow batteries: Status and perspective towards sustainable stationary energy storage. J. Power Sources, 2021, 481, 228804.

${ }^{6}$ L. Trahey, F. R. Brushett, N. P. Balsara, G. Ceder, L. Cheng, Y. M. Chiang, N. T. Hahn, B. J. Ingram, S. D. Minteer, J. S. Moore, K. T. Mueller, L. F. Nazar, K. A. Persson, D. J. Siegel, K. Xu, K. R. Zavadil, V. Srinivasan, and G. W. Crabtree, Energy storage emerging: A perspective from the Joint Center for Energy Storage Research. Proc. Natl. Acad. Sci. U. S. A. 2020, 117, 12550-12557.

7 B. Hu, J. Luo, C. DeBruler, M. Hu, W. Wu, and T. L. Liu, Redoxactive inorganic materials for redox flow batteries. Encyclopedia of Inorganic and Bioinorganic Chemistry, 2019 by John Wiley and Sons, Ltd.

${ }^{8}$ K. Lourenssen, J. Williams, F. Ahmadpour, R. Clemmer, and S. Tasnim, Vanadium redox flow batteries: A comprehensive review. J. Energy Storage, 2019, 25, 100844.

${ }^{9} \mathrm{C}$. Minke and T. Turek, Materials, system designs and modelling approaches in techno-economic assessment of all-vanadium redox flow batters. J. Power Sources, 2018, 376, 66-81.

$10 \mathrm{C}$. Sun and H. Zhang, Review of the development of firstgeneration redox flow batteries: Iron-chromium system. ChemSusChem, 2021, 14, ASAP. DOI: 10.1002/cssc. 202101798

${ }_{11}$ A. Khor, P. Leung, M. R. Mohamed, C. Flox, Q. Xu, L. An, R. G. A. Wills, J. R. Morante, and A. A. Shah, Review of zinc-based hybrid flow batteries: from fundamentals to applications. Mater. Today Energy, 2018, 8, 80-108.

12 R. M. Darling, K. G. Gallagher, J. A. Kowalski, S. Ha, and F. R. Brushett, Pathways to low-cost electrochemical energy storage: a comparison of aqueous and nonaqueous flow batteries. Energy Environ. Sci., 2014, 7, 3459-3477.

${ }^{13}$ V. Viswanathan, A. Crawford, D. Stephenson, S. Kim, W. Wang, B. Li, G. Coffey, E. Thomsen, G. Graff, P. Balducci, M. KintnerMeyer, and V. Sprenkle, Cost and performance model for redox flow batteries. J. Power Sources, 2014, 247, 1040-1051.

${ }_{14} \mathrm{M}$. Li, Z. Rhodes, J. R. Cabrera-Pardo, and S. D. Minteer, Recent advancements in rational design of non-aqueous organic redox flow batteries. Sustain. Energy Fuels, 2020, 4, 4370-4389.
${ }^{15} \mathrm{M}$. Li, J. Case, and S. D. Minteer, Bipolar redox-active molecules in non-aqueous organic redox flow batteries: Status and challenges. ChemElectroChem, 2021, 8, 1215-1232.

16 Y. Ding, C. Zhang, L. Zhang, Y. Zhou, and G. Yu, Molecular engineering of organic electroactive materials for redox flow batteries. Chem. Soc. Rev., 2018, 47, 69-103

$17 \mathrm{~J}$. Luo, B. Hu, M. Hu, Y. Zhao, and T. L. Liu, Status and prospects of organic redox flow batteries toward sustainable energy storage. ACS Energy Lett., 2019, 4, 2220-2240.

${ }^{18} \mathrm{~B}$. Hu and T. L. Liu, Two electron utilization of methyl viologen anolyte in nonaqueous organic redox flow battery. J. Energy Chem., 2018, 27, 1326-1332.

${ }^{19}$ C. Chen, S. Zhang, Y. Zhu, Y. Qian, Z. Niu, J. Ye, Y. Zhao, and X. Zhang, Pyridyl group design in viologens for anolyte materials in organic redox flow batteries. RSC Adv., 2018, 8, 18762-18770.

20 J. Chai, A. Lashgari, Z. Cao, C. K. Williams, X. Wang, J. Dong, and J. Jiang, PEGylation-enabled extended cyclability of a nonaqueous redox flow battery. ACS Appl. Mater. Interfaces, 2020, 12, 15262-15270.

21 J. Chai, A. Lashgari, X. Wang, C. K. Williams, and J. Jiang, AllPEGylated redox-active metal-free organic molecules in nonaqueous redox flow battery. J. Mater. Chem. A, 2020, 8, 1571515724.

22 N. H. Attanayake, Z. Liang, Y. Wang, A. P. Kaur, S. R. Parkin, J. K. Mobley, R. H. Ewoldt, J. Landon, and S. A. Odom, Dual function organic active materials for nonaqueous redox flow batteries. Mater. Adv., 2021, 2, 1390-1401.

${ }^{23}$ M. Kathiresan, B. Ambrose, N. Angulakshmi, D. E. Mathew, D. Sujatha, and A. M. Stephan. Viologens: a versatile organic molecule for energy storage applications. J. Mater. Chem. A, 2021, 9, 27215-27233.

24 Z. Li, S. Li, S. Liu, K. Huang, D. Fang, F. Wang, and S. Peng, Electrochemical properties of an all-organic redox flow battery using 2,2,6,6-tetramethyl-1-piperidinyloxy and Nmethylphthalimide. Electrochem. Solid-State Lett., 2011, 14, A171-A173.

$25 \mathrm{~J}$. Winsberg, S. Benndorf, A. Wild, M. D. Hager, and U. S. Schubert, Synthesis and characterization of a phthalimidecontaining redox-active polymer for high-voltage polymer-based redox-flow batteries. Macromol. Chem. Phys., 2018, 219, 1700267.

${ }^{26}$ H.-S. Kim, S. Hwang, Y. Kim, J. H. Ryu, S. M. Oh, and K. J. Kim, Bifunctional effects of lengthening aliphatic chain of phthalimidebased negative redox couple and its non-aqueous flow battery performance at stack cell. APL Materials, 2018, 6, 047901.

${ }^{27}$ C. Zhang, Z. Nui, Y. Ding, L. Zhang, Y. Zhou, X. Guo, X. Zhang, Y. Zhao, and G. Yu, Highly concentrated phthalimide-based anolytes for organic redox flow batteries with enhanced reversibility. Chem, 2018, 4, 2814-2825.

28 S. Hwang, H.-S. Kim, J. H. Ryu, and S. M. Oh, NFerrocenylphthalimide; A single redox couple formed by attaching a ferrocene moiety to phthalimide for non-aqueous flow batteries. J. Power Sources, 2018, 395, 60-65. 
29 N. Daub, R. A. J. Janssen, and K. H. Hendriks, Imide-based multielectron anolytes as high-performance materials in nonaqueous redox flow batteries. ACS Appl. Energy Mater., 2021, 4, 9248-9257.

${ }^{30}$ D. Xu, C. Zhang, Y. Zhen, and Y. Li, Ferrocene/phthalimide ionic bipolar redox-active molecule for symmetric nonaqueous redox flow batteries. ACS Appl. Energy Mater., 2021, 4, 8045-8051.

${ }^{31}$ W. Duan, J. Huang, J. A. Kowalski, I. A. Shkrob, M. Vijayakumar, E. Walter, B. Pan, Z. Yang, J. D. Milshtein, B. Li, C. Liao, Z. Zhang, W. Wang, J. Liu, J. S. Moore, F. R. Brushett, L. Zhang, and X. Wei, "Wine-dark sea" in an organic flow battery: storing negative charge in 2,1,3-benzothiadiazole radicals leads to improved cyclability. ACS Energy Lett., 2017, 2, 1156-1161.

32 J. Zhang, J. Huang, L. A. Robertson, R. S. Assary, I. A. Shkrob, and L. Zhang, Elucidating factors controlling long-term stability of radical anions for negative charge storage in nonaqueous redox flow batteries. J. Phys. Chem. C, 2018, 122, 8116-8127.

33 Y. Zhao, E. S. Sarnello, L. A. Robertson, J. Zhang, Z. Shi, Z. Yu, S R. Bheemireddy, Y Z, T. Li, R. S. Assary, L. Cheng, Z. Zhang, L. Zhang, and I. A. Shkrob, Competitive pi-stacking and h-bond piling increase solubility of heterocyclic redoxmers. J. Phys. Chem. B, 2020, 124, 10409-10418.

${ }^{34}$ L. A. Robertson, I. A. Shkrob, G. Agarwal, Y. Zhao, Z. Yu, R. S. Assary, L. cheng, J. S. Moore, and L. Zhang Fluorescence-enabled self-reporting for redox flow batteries. ACS Energy Lett., 2020, 5 3062-2068.

${ }^{35}$ C. S. Sevov, R. E. M. Brooner, E. Chénard, R. A. Assary, J. S. Moore, J. Rodríguez-Loṕez, and M. S. Sanford, Evolutionary design of low molecular weight organic anolyte materials for applications in nonaqueous redox flow batteries. J. Am. Chem. Soc., 2015, 137, 14465-14472.

36 C. S. Sevov, K. H. Hendriks, and M. S. Sanford, Low-potential pyridinium anolytes for aqueous redox flow batteries. J. Phys. Chem. C, 2017, 121, 24376-24380.

${ }^{37}$ C. S. Sevov, D. P. Hickey, M. E. Cook, S. G. Robinson, S. Barnett, S. D. Minteer, M. S. Sigman, and M. S. Sanford, Physical organic approach to persistent, cyclable, low-potential electrolytes for flow battery applications. J. Am. Chem. Soc., 2017, 139, 29242927.

38 K. H. Hendriks, C. S. Sevov, M. E. Cook, and M. S. Sanford, Multielectron cycling of a low-potential anolyte in alkali meta electrolytes for nonaqueous redox flow batteries. ACS Energy Lett., 2017, 2, 2430-2435.

${ }^{39}$ P. W. Antoni, T. Bruckhoff, and M. M. Hansmann, Organic redox systems based on pyridinium-carbene hybrids. J. Am. Chem. Soc., 2019, 141, 9701-9711.

${ }^{40}$ S. Ahn, J. H. Jang, J. Kang, M. Na, J. Seo, V. Singh, J. M. Joo, and $H$. R. Byon, Systematic designs of dicationic heteroarylpyridiniums as negolytes for nonaqueous redox flow batteries. ACS Energy Lett., 2021, 6, 3390-3397.

41 J. D. Griffin, A. R. Pancoast, and M. S. Sigman, Interrogation of 2,2'-bipyrimidines as low-potential two-electron electrolytes. J. Am. Chem. Soc., 2021, 143, 992-1004.

42 J. Zhang, J. Huang, L. A. Robertson, I. A. Shkrob, and L. Zhang, Comparing calendar life and cycle life stability of redox active organic molecules for nonaqueous redox flow batteries. J. Power Sources, 2018, 397, 214-222.

${ }^{43}$ C. A. Hunter and J. K. M. Sanders, The nature of $\pi-\pi$ interactions. J. Am. Chem. Soc., 1990, 112, 5255-5534.

${ }^{44} \mathrm{~F}$. Bertinotti, C. Giacomello, and A. M. Liquori, The structure of heterocyclic compounds containing nitrogen. I. Crystal and molecular structure of s-tetrazine. Acta Cryst., 1956, 9, 510-514.
45 M. Yu. Antipin, T. V. Timofeeva, D. S. Yufit, and J. Sauer, Molecular structure and electron density distribution in the crystals of 3,6-dimethoxy-1,2,4,5-tetrazine and 3-phenyl-1,2,4,5tetrazine based on X-ray diffraction data at 120 and $150 \mathrm{~K}$. "Bent" bonds in the six-membered heterocycle. Russ. Chem. Bull., 1995, 44, 2337-2345.

${ }^{46}$ E. S. Salmina, P. A. Slepukhin, G. L. Rusinov, M. A. Grishina, and V. A. Potemkin, Stacking interactions in homomolecular crystals formed by organic compounds containing s-tetrazine moiety, Butlerov Comm., 2011, 26, 19-31.

$47 \mathrm{G}$. Clavier and P. Audebert, $s$-Tetrazines as building blocks for new functional molecules and molecular materials. Chem. Rev., 2010, 110, 3299-3314.

48 Y. Kim, J. Do, E. Kim, G. Clavier, L. Galmiche, and P. Audebert, Tetrazine-based electrofluorochromic windows: modulation of the fluorescence through applied potential. J. Electroanal. Chem., 2009, 632, 201-205.

49 F. Miomandre and P. Audebert, 1,2,4,5-Tetrazines: An intriguing heterocycles family with outstanding characteristics in the field of luminescence and electrochemistry. J. Photochem. Photobio. C: Photochem. Rev., 2020, 44, 100372.

$50 \mathrm{~S}$. Samanta, S. Das, and P. Biswas, Photocatalysis by 3,6disubstituted-s-tetrazine: visible-light driven metal-free green synthesis of 2-substituted benzimidazole and benzothiazole. J. Org. Chem., 2013, 78, 11184-11193.

51 S. Samanta and P. Biswas, Metal free visible light driven oxidation of alcohols to carbonyl derivatives using 3,6-di(pyridine2-yl)-1,2,4,5-tetrazine (pytz) as catalyst. RSC Adv., 2015, 5, 8432884333.

52 T. Le, T. Courant, J. Merad, C. Allain, P. Audebert, and G. Masson, s-Tetrazine dyes: A facile generation of photoredox organocatalysts for routine oxidations. J. Org. Chem., 2019, 84, 16139-16146.

$53 \mathrm{~J}$. Lyu, T. Le, A. Claraz, C. Allain, P. Audebert, and G. Masson, sTetrazine: Robust and green photoorganocatalyst for aerobic oxidation of $\mathrm{N}, \mathrm{N}$-disubstituted hydroxylamines to nitrones. Synlett, 2021, 32, ASAP. DOI: 10.1055/a-1691-0449

54 W.-K. An, S.-J. Zheng, H.-X. Zhang, T.-T. Shang, H.-R. Wang, X.-J. Xu, Q. Jin, Y. Qin, Y. Ren, S. Jiang, C.-L. Xu, M.-S. Hou, and Z. Pan, $s$-Tetrazine-functionalized hyper-crosslinked polymers for efficient photocatalytic synthesis of benzimidazoles. Green Chem., 2021, 23, 1292-1299.

${ }^{5}$ D. J. Min, F. Miomandre, P. Audebert, J. E. Kwon, and S. Y. Park, $s$-Tetrazines as a new electrode-active material for secondary batteries. ChemSusChem, 2019, 12, 503-510.

56 D. J. Min, K. Lee, H. Park, J. E. Kwon, and S. Y. Park, Redox potential tuning of $s$-tetrazine by substitution of electronwithdrawing/donating groups for organic electrode materials. Molecules, 2021, 26, 894.

${ }^{57}$ R. M. Versteegen, R. Rossin, W. ten Hoeve, H. M. Janssen, and M. S. Robillard, Click to release: Instantaneous doxorubicin elimination upon tetrazine ligation. Angew. Chem. Int. Ed., 2013, 52, 14112-14116.

58 P. Audebert, F. Miomandre, G. Clavier, M.-C. Vernières, S. Badré, and R. Méallet-Renault, Synthesis and properties of new tetrazines substituted by heteroatoms: Towards the world's smallest organic fluorophores. Chem. Eur. J., 2005, 11, 56675673.

59 Y. H. Gong, F. Miomandre, R. Méallet-Renault, S. Badré, L. Galmiche, J. Tang, P. Audebert, and G. Clavier, Synthesis and physical chemistry of $s$-tetrazines: which ones are fluorescent and why? Eur. J. Org. Chem., 2009, 35, 6121-6128.

60 B. J. Jordan, M. A. Pollier, L. A. Miller, C. Tiernan, G. Clavier, P. Audebert, and V. M. Rotello, Redox-modulated recognition of tetrazines using thioureas. Org. Lett., 2007, 9, 2835-2838.

61 A. J. Bard and L. R. Faulkner, Electrochemical Methods: Fundamentals and Applications; Harris, D.; Swain, E.; Robey C.; Aiello, E., John Wiley and Sons, INC.: US, 2001; pp. 230-243. 
62 X. Wei, W. Duan, J. Huang, L. Zhang, B. Li, D. Reed,W. Xu, V. Sprenkle, and W. Wang, A high-current, stable nonaqueous organic redox flow battery. ACS Energy Lett., 2016, 1, 705-711.

63 I. Lavagnini, R. Antiochia, and F. Magno, An extended method for the practical evaluation of the standard rate constant from cyclic voltammetric data. Electroanalysis, 2004, 16, 505-506.

64 A brown solid was observed during the Tz-Me cycling experiments, suggesting either precipitation of the insoluble radical anion or chemical degradation.

${ }^{65} \mathrm{~K}$. Izutsu, Properties of solvents and solvent classification. In Electrochemistry in nonaqueous solution, second edition. WILEYVCH Verlag GmbH \& Co. KGaA, US, 2009, pp. 1-25. 